\title{
Adaptación y validación española del Adolescent-Cannabis Problems Questionnaire (CPQ-A)
}

\section{Spanish adaptation and validation of The Adolescent- Cannabis Problems Questionnaire (CPQ-A)}

\author{
Sergio Fernández-ArtamendI*; José Ramón \\ Fernández-HermidA*; EduARdo García-Cueto*; \\ Roberto Secades-Villa*; Gloria García-Fernández*; \\ SILVIA BARRIAL-BARBÉN ${ }^{\star *}$
}

* Grupo de Investigación sobre Conductas.

Adictivas, Universidad de Oviedo.

** Facultad de Psicología, Universidad de Oviedo.

Enviar correspondencia a:

Sergio Fernández Artamendi.

Grupo de Investigación en Conductas Adictivas.

Universidad de Oviedo. Plaza Feijoo s/n, 33003 - Oviedo (Asturias).

E-mail: fernandezasergio@uniovi.es TIf: 0034985104189.

\section{Resumen}

El cannabis es una sustancia cuyo consumo está altamente extendido entre jóvenes y adolescentes. Sin embargo, la demanda de ayuda y tratamiento en esas edades sigue siendo baja. En estas condiciones resulta esencial el desarrollo de instrumentos especialmente diseñados para la detección precoz de los problemas asociados al consumo de cannabis en esa población. El propósito de este trabajo es adaptar y validar en población española el CPO-A (AdolescentCannabis Problems Questionnaire) como herramienta de screening. La muestra utilizada está formada por 144 jóvenes (71,4\% chicos), con edades comprendidas entre 16 y 20 años $(M=17,12 ; D T=1,17)$, que habian consumido cannabis en el último mes, y que se encontraban cursando algún tipo de formación en diversos centros educativos de Asturias. El análisis factorial del CPO-A en esta muestra apunta a una solución unidimensional, con un Alfa de Cronbach de 0,86 para el total del cuestionario. Las evidencias de validez convergente de la prueba son buenas. La puntuación en el CPO-A permite discriminar entre consumidores de cannabis moderados y excesivos en el último mes, está positivamente relacionada con el CAST (Cannabis Abuse Screening Test) y presenta una sensibilidad y especificidad adecuadas para determinar casos de abuso y dependencia del cannabis según los criterios del DSM IV-TR. Además, es sensible al grado de preocupación que genera en los jóvenes el consumo de cannabis y los efectos psicopatológicos asociados al consumo. Los resultados obtenidos permiten afirmar que es una prueba útil para el screening de jóvenes consumidores de cannabis con problemas.

Palabras clave: cannabis, screening, evaluación psicométrica, jóvenes, adolescentes.

\section{Abstract}

Cannabis is a widely used substance among adolescents and young adults, but demand for help and treatment from this age group continues to be low. It is therefore essential to develop instruments especially designed for the early detection of problems associated with cannabis use in this population. The purpose of this work is to adapt and validate the CPO-A (Adolescent Cannabis Problems Questionnaire) for its use as a screening instrument in Spanish population. The sample was made up of 144 young people ( $71.5 \%$ male) aged 16 to $20(M=17.12 ; S D=1.17)$ who had used cannabis in the last month and were on some kind of study or training course at various educational institutions in Asturias (a region in northern Spain). Factor analysis of the CPO-A in this sample provides evidence of a unidimensional structure, with a Cronbach's Alpha of 0.86 for the questionnaire. The evidence also shows that the instrument's convergent validity is good. The CPO-A score permits discrimination between moderate and excessive cannabis users over the last month, is positively related to score on the CAST (Cannabis Abuse Screening Test), and presents adequate sensitivity and specificity for identifying cases of cannabis abuse and dependence according to the DSM IV-TR criteria. It is also sensitive to young people's degree of concern about cannabis use and the psychopathological effects associated with it. In accordance with the results obtained, the CPO-A can be considered a useful screening tool for young cannabis users with problems.

Key words: cannabis, marijuana, screening, psychometrics, young people. 
D e acuerdo con la Encuesta Nacional sobre el uso de Drogas y la Salud (NSDUH), en el 2009 16,7 millones de estadounidenses mayores de 12 años habian consumido cannabis por lo menos una vez en el mes previo a la encuesta. Esta prevalencia representa el 6,6\% de la población mayor de 12 años, y el 76,6\% de todos los que consumen sustancias ilegales (Substance Abuse and Mental Health Services Administration, 2010). No existe información estadistica actualizada de toda la Unión Europea, pero los datos más recientes de prevalencia de consumo en Europa en el último mes indican que hay fuertes diferencias entre países, con cifras que oscilan entre el 1,6 y el 7,6\% de las personas de 15 a 64 años según el European Monitoring Centre for Drugs and Drug Addiction (2011). España supera la media europea y se sitúa en la parte superior de la distribución, con una prevalencia de 7,2\% en el 2007 (último año del que se dispone de datos), de personas entre 15 y 64 años que han consumido cannabis en el último mes (Observatorio Español sobre Drogas, 2009).

Estos datos son preocupantes teniendo en cuenta los riesgos del consumo de cannabis para la salud mental (Fernández-Artamendi; Fernández-Hermida; Secades-Villa y Garcia-Portilla, 2011). Su uso se ha relacionado con deterioros cognitivos observables en numerosas funciones ejecutivas como la capacidad para planificar, resolver problemas, tomar decisiones, así como recordar y controlar las emociones y la conducta (Crean, Crane, y Mason, 2011). Consumirlo de forma habitual se ha asociado también con la realización de conductas de riesgo (Baño Rodrigo et al., 2004) y con una mayor probabilidad de padecer trastornos psicopatológicos como depresión, síndrome amotivacional y trastornos psicóticos (Rubino, Zamberletti, y Parolaro, 2011). La literatura actual no considera el cannabis como una sustancia inocua, sino que los estudios epidemiológicos proponen el consumo de cannabis como factor de riesgo para otros problemas de salud mental (Arias Horcajadas, 2007; Hall y Degenhardt, 2009; Parolaro, 2010).

La alta prevalencia de consumo de cannabis junto con la peligrosidad asociada a su consumo hacen aconsejables la detección e intervención precoces, cuando el hábito está menos instaurado y aún no ha generado graves consecuencias. Lamentablemente, la detección precoz es difícil ya que los consumidores jóvenes no suelen solicitar ayuda (Caldeira et al., 2009). De hecho, en España sólo el 0,73\% de los jóvenes que usan cannabis han recibido tratamiento en alguna ocasión (Observatorio Español sobre Drogas, 2009). Parece existir una baja percepción de riesgo relacionada con el uso esporádico o continuado de esta substancia (Observatorio Español sobre Drogas, 2009), por lo que tanto los usuarios como su entorno no consideran necesariamente que su consumo pueda constituir un problema. Es habitual que los consumidores que buscan ayuda profesional lo hagan tras una amplia historia de consumo (Kessler et al., 2001).

El uso de instrumentos de screening, breves y fiables, que se puedan aplicar a amplios sectores de jóvenes escolarizados, es esencial en esta situación. Dadas las limitaciones del uso de las categorías de abuso y dependencia del DSM-IV (Diagnostic and Statistical Manual) con este tipo de pobla- ción joven (Hartman et al., 2008), los instrumentos de screening se centran fundamentalmente en la detección de los problemas más comúnmente asociados al uso del cannabis. Su utilidad fundamental consiste en discriminar entre usuarios experimentadores y aquéllos que ya tienen un consumo problemático y que son susceptibles de beneficiarse de una intervención precoz. En estas pruebas se evalúan las alteraciones de la salud física o psíquica, los problemas sociales y de rendimiento, e incluso conductas de riesgo que pueden poner en peligro la vida o la salud del consumidor.

En la literatura internacional se encuentran diferentes instrumentos para la evaluación de los problemas derivados del consumo de cannabis. Entre ellos se encuentra el CAST - Cannabis Abuse Screening Test - (Legleye, Karila, Beck, y Reynaud, 2007), el SDS - Severity of Dependence Scale (Gossop et al., 1995), el CUDIT-R - Cannabis Use Disorder Identification Test - Revised - (Adamson et al., 2010) y el CPO-A (Martin, Copeland, Gilmour, Gates, y Swift, 2006). No obstante, únicamente el CAST y el CPQ-A son cuestionarios elaborados específicamente para la evaluación de los problemas generados por el consumo de cannabis en jóvenes y adolescentes. De ellos, tan sólo el CAST se encuentra validado en jóvenes consumidores de cannabis en España (Klempova et al., 2009).

EI CAST es un instrumento de 6 ítems diseñado para detectar patrones de abuso de cannabis en jóvenes y adolescentes, centrándose sobre todo en las dificultades para controlar el consumo y en sus consecuencias negativas sobre la salud o las relaciones sociales (Piontek, Kraus y Klempova, 2008). El formato de respuesta es tipo Likert en función de la frecuencia de experimentación de cada uno de los problemas descritos (de 1 = nunca a 5 = muy a menudo), oscilando las puntuaciones finales entre 6 y 30 . Un estudio reciente ha realizado una primera validación en España de este instrumento (Klempova et al., 2009) con 3569 participantes jóvenes, obteniendo una buena fiabilidad (Alfa de Cronbach $=0,72$ ).

El Adolescent Cannabis Problems Questionnaire (CPQ-A) consta de 27 items y es una adaptación del Cannabis Problems Questionnaire - CPO - (Copeland, Gilmour, Gates, y Swift, 2005). El CPQ-A es una prueba desarrollada para la detección de los problemas que se asocian más comúnmente con el consumo de cannabis en jóvenes. Según los resultados del estudio de Copeland et al. (2005), dichos problemas parecen agruparse en tres factores, altamente interrelacionados: consecuencias psicológicas/financieras, consecuencias físicas y consecuencias negativas agudas del consumo de cannabis. La prueba ha demostrado ser una medida fiable (con alfas de Cronbach entre 0,72 y 0,88 para cada uno de los factores) de los problemas relacionados con el consumo de cannabis. Ofrece además altas correlaciones test-retest $(r=0,91)$, siendo los resultados comparables al CPO para adultos (Martin et al., 2006).

El CPQ-A fue creado como herramienta de apoyo a la evaluación clínica. Sin embargo, dadas sus buenas propiedades psicométricas, su facilidad de aplicación y relativa brevedad, unido a la efectividad para detectar problemas asociados al consumo de cannabis en jóvenes y adolescen- 
tes hacen interesante su adaptación como instrumento de screening en población española.

El objetivo de esta investigación es adaptar y validar el CPQ-A en una muestra de jóvenes españoles. Se pretende conocer el comportamiento de la prueba como medida de los problemas asociados al consumo de cannabis, de forma que se facilite el screening de las situaciones de consumo no experimental y una posible intervención precoz. Se parte de la hipótesis de que el instrumento mantendrá las buenas propiedades psicométricas de fiabilidad y validez obtenidas en anteriores estudios. Se espera encontrar variaciones en la estructura factorial, dadas las diferencias culturales y en las características de la muestra respecto al estudio inicial de Copeland et al. (2005).

\section{Método}

\section{Participantes}

La muestra inicial estaba compuesta por 1.000 estudiantes de 9 centros escolares de Asturias, pertenecientes a los niveles educativos de bachillerato y formación profesional. Los centros escolares se seleccionaron en aquéllas áreas con mayores prevalencias de consumo, con el fin de garantizar el máximo número de consumidores de cannabis. La participación en el estudio era voluntaria y contaba con la aprobación previa del centro y de las autoridades educativas. Ningún estudiante renunció a participar en el estudio.

Las respuestas obtenidas fueron filtradas mediante un cuestionario de infrecuencia, que eliminaba las contestaciones dadas al azar, tal y como se detalla más abajo, obteniéndose en total 878 respuestas válidas. De éstas, 130 fueron descartadas por pertenecer a participantes con una edad situada fuera del rango del estudio. La muestra resultante estaba constituida por 748 estudiantes de entre 16 y 20 años $(M=17,12 ; D T=1,17)$. Finalmente, la muestra final para este trabajo se limitó a aquellos que habian consumido cannabis en el último mes y que, por lo tanto, podían completar el CPQ-A y el resto de instrumentos dirigidos a evaluar las consecuencias del consumo. Un total de 144 participantes completaron el CPQ-A, con una edad media de 17,38 años $(D T=1,16)$, de los cuales un $71,5 \%$ eran chicos y un $28,5 \%$ eran chicas.

Se estimó que una muestra de 144 participantes sería adecuada, ya que con una varianza de 16,893 que se obtiene en el test, al Nivel de Confianza del $95 \%$, se trabaja con un error $\leq 0,67$.

\section{Procedimiento}

La traducción del CPQ-A ha buscado garantizar la equivalencia idiomática de los contenidos de las versiones en inglés y español, de acuerdo con lo que se indica en las Directrices para la Traducción y Adaptación de los Tests de la International Test Commission (Muñiz-Fernández y Ham- bleton, 1996). Para ello se realizó tanto una traducción como una retro-traducción del español al inglés, esta última a cargo de un experto traductor anglo-parlante.

El CPQ-A ya traducido (Ver Anexo 1), se aplicó junto con una batería de pruebas mediante un procedimiento computerizado elaborado con el software LimeSurvey ${ }^{\circledR}$. El programa presentaba a los participantes únicamente aquellas secciones de la encuesta aplicables a su situación personal en función de la información que aportaban, alertándoles para corregir incongruencias e impidiendo que se generasen encuestas incompletas y valores perdidos. Se garantizó la confidencialidad y el anonimato de las respuestas mediante la utilización de una base de datos en red con la información recogida en la encuesta, que no solicitaba información personal identificativa. El estudio fue realizado con la aprobación de la Consejería de Educación del Principado de Asturias, cumpliendo todos los requisitos éticos. La realización de la encuesta fue supervisada por psicólogos profesionales entrenados en el uso de los instrumentos utilizados.

\section{Medidas}

La encuesta aplicada constó de los siguientes sub-apartados:

Información sociodemográfica. Se solicitó a los participantes que aportaran información sobre su edad, sexo y estatus de inmigrante (nacional o extranjero).

Consumo de sustancias. La frecuencia y los patrones de consumo de sustancias fueron evaluados mediante items del European School Survey Project on Alcohol and Other Drugs Student Questionnaire 2007 - ESPAD - (ESPAD Project, 2009), con preguntas que recogian información sobre la prevalencia de consumo de cannabis así como de tabaco, alcohol y otras drogas ilegales durante la última semana, el último mes, el último año y toda la vida. También se preguntó por la frecuencia con la que habian llegado a casa bajo los efectos del cannabis en los mismos periodos de tiempo. Las posibilidades de respuesta para cada periodo temporal incluian: $1-2$ veces, $3-5$ veces, $6-9$ veces, $10-19$ veces, $20-39$ veces y 40 o más ocasiones.

Evaluación de los problemas por consumo de cannabis. La evaluación de los problemas asociados al consumo de cannabis se realizó desde diversas perspectivas: Además del cuestionario objeto de validación, se aplicó el CAST, y se evaluó la presencia de síntomas y cumplimiento de criterios de abuso y dependencia según el DSM-IV-TR (American Psychiatric Association, 2002) así como la preocupación de los participantes por los problemas ocasionados por el consumo de cannabis. El objetivo era obtener diversas medidas de los problemas derivados del consumo que facilitaran el estudio de la validez convergente del CPO-A.

En primer lugar, la presencia de abuso y dependencia del cannabis se evaluó a través de dos conjuntos de preguntas derivadas de los correspondientes criterios del DSM-IV-TR para abuso y dependencia (American Psychiatric Association, 2002). La presencia de síntomas de esos trastornos indica un 
uso problemático que se distingue del uso ocasional o experimental, por lo que se hipotetiza que debe guardar relación con las puntuaciones obtenidas en el CPO-A.

En segundo lugar, se aplicó la prueba objeto de adaptación y validación, el CPO-A (ver Anexo). Como instrumento para la validación externa, se aplicó también el CAST, un instrumento que ha mostrado una buena fiabilidad (Alfa de Cronbach $=0,72$ ) en población española. Estos dos tests autoaplicados valoran directamente la presencia de problemas comúnmente asociados al consumo de cannabis en población joven.

Por último, se valoró el nivel de preocupación general producido como consecuencia del consumo de cannabis. Para ello se utilizó una escala Likert de 0 (ninguna preocupación) a 10 (muy alta preocupación). En este apartado, además, se investigaron los ámbitos específicos a los que podía afectar dicha preocupación (rendimiento académico, relacionas familiares, relación de pareja, relaciones con amigos, memoria, rendimiento laboral, salud, futuro, consumo de otras sustancias, perder la capacidad de divertirse sin drogas) mediante la formulación de preguntas dicotómicas. Se parte de la hipótesis de que una mayor preocupación y un mayor número de áreas afectadas se asociarán con una mayor puntuación en el CPQ-A.

Sintomatología psicopatológica. Se aplicó el Brief Symptom Inventory - BSI - (Derogatis y Melisaratos, 1983) para evaluar la afectación psicopatológica. Este instrumento obtiene puntuaciones en las siguientes 9 dimensiones: somatización, obsesivo-compulsiva, sensibilidad interpersonal, depresión, ansiedad, hostilidad, ansiedad fóbica, ideación paranoide y psicoticismo. Ofrece además un Índice de Severidad Global, un Índice de Malestar por Síntomas Positivos y un Total de Sintomas Positivos. La fiabilidad de cada una de las dimensiones de la prueba en muestra española oscila entre 0,72 y 0,95 (Pereda, Forns y Pero, 2007).

Escala de infrecuencia. A lo largo de la encuesta se intercaló una escala de infrecuencia con el objetivo de detectar aquellos cuestionarios que habian sido respondidos de forma aleatoria o errática. El instrumento seleccionado fue la Escala Oviedo de Infrecuencia (Fonseca-Pedrero, Paino-Piñeiro, Lemos-Giráldez, Villazón-Garcia, y Muñiz, 2009), que evalúa el acuerdo o desacuerdo con 12 ítems del tipo "se llega antes de Madrid a Moscú en coche que en avión", con un formato de respuesta tipo Likert (de 1 = muy en desacuerdo a 5: muy de acuerdo). Los cuestionarios con más de tres respuestas erróneas no fueron considerados para posteriores análisis, siguiendo las instrucciones establecidas por los autores.

\section{Análisis de datos}

En primer lugar se realizó un análisis descriptivo de las variables sociodemográficas y referentes al consumo de cannabis, con el fin de determinar la similitud de los resultados obtenidos en nuestra muestra con los de la población nacional, y la posible generalización de resultados.
A continuación se realizó un análisis descriptivo de cada uno de los ítems que componen el cuestionario, para proporcionar un análisis detallado de los resultados obtenidos en el cuestionario por parte de los participantes. Para ello se incluyeron en el análisis aquellos cuestionarios cuya puntuación era superior a cero, con el fin de evitar sesgos en los datos obtenidos.

Para determinar la semejanza entre la estructura interna de la versión española y la versión original del CPQ-A se llevó a cabo un análisis factorial confirmatorio. Para la estimación se utilizó el método de Máxima Verosimilitud. A continuación se sometieron los datos obtenidos en la aplicación del test a diversos análisis factoriales exploratorios con la finalidad de averiguar la mejor estructura del test para la versión española. Finalmente, se realizó un análisis factorial confirmatorio para validar la estructura propuesta. Se llevaron a cabo por tanto análisis factoriales confirmatorios y exploratorios a nivel de los ítems de la escala para comprobar su estructura factorial mediante el screeplot, índices de discriminación, pesos factoriales y porcentaje de varianza explicada.

Las evidencias de validez predictiva concurrente de la escala se investigaron a través de cuatro análisis. Primeramente se calculó la correlación de la puntuación total en el CPQ-A con la intensidad del consumo en el último mes. Además, se halló la odds ratio de la puntuación global del CPO-A sobre la intensidad de consumo en el último mes. Para ello, se dividió a los consumidores en dos grupos: Consumo Moderado -aquellos con un consumo inferior a 6 ocasiones en el último mes; y Consumo Elevado -aquellos que refieren 6 o más ocasiones de consumo en el último mes. En segundo lugar se calculó la correlación de Pearson del CPQ-A con el CAST. En tercer lugar, se realizó una regresión logística de las puntuaciones globales del CPO-A respecto a los criterios de abuso y dependencia del DMS-IV-TR. A continuación se calculó también, mediante la curva ROC, el punto de corte óptimo de la escala para maximizar la capacidad del instrumento de detectar a los consumidores con abuso o dependencia del cannabis según el DSM-IV (sensibilidad) y de discriminar a aquellos sin diagnóstico (especificidad). Por último, se analizó mediante la prueba t de Student si la aplicación de los puntos de corte óptimos hallados para la dependencia permitían detectar a aquellos participantes con puntuaciones superiores en sintomatología psicopatológica, evaluada según el BSI. Se calculó la d de Cohen para estimar el tamaño del efecto de dichas diferencias.

Para los análisis se utilizó el SPSS para Windows, versión 15, excepto para los análisis factoriales confirmatorios, que se realizaron con el AMOS 16, y la d de Cohen que se calculó de acuerdo con la siguiente fórmula: 


\section{Resultados}

\section{Datos sociodemográficos y de consumo}

Los resultados obtenidos en este apartado pueden verse en la Tabla 1. En dicha tabla se comparan los resultados de la muestra actual con los últimos de ámbito nacional de la encuesta ESTUDES, realizada en centros escolares, y publicados por el Plan Nacional sobre Drogas (Observatorio Español sobre Drogas, 2009).

Tabla 1. Datos sociodemográficos y de consumo de cannabis

\begin{tabular}{lcccc}
\hline \multirow{2}{*}{ Variable } & \multicolumn{4}{c}{ Estudio } \\
\cline { 2 - 5 } & \multicolumn{3}{c}{ ESTUDES - PNSD } & \multicolumn{2}{c}{ Muestra actual } \\
\hline \multirow{2}{*}{ Edad } & $\begin{array}{l}\text { M }=\text { No disponible. } \\
\text { Rango: 14-18. }\end{array}$ & \multicolumn{2}{c}{$\begin{array}{l}\text { M=17,12 } \\
\text { Rango: 16-20 }\end{array}$} \\
\hline & Hombres & Mujeres & Hombres & Mujeres \\
\hline Sexo (\%) & 49,53 & 50,47 & 59,3 & 40,7 \\
Edad inicio consumo cannabis (M) & 14,6 & 14,7 & 15,5 & 15,5 \\
Prevalencia consumo alguna vez en la vida (\%) & 37,8 & 32,8 & 44,7 & 33,2 \\
Prevalencia consumo últimos 12 meses (\%) & 33,5 & 27,5 & 37,0 & 27,7 \\
Prevalencia consumo último mes (\%) & 23,0 & 17,2 & 23,5 & 13,2 \\
\hline
\end{tabular}

\section{Estadísticos descriptivos}

En la Tabla 2 se incluyen los principales estadísticos descriptivos del cuestionario CPQ-A. Los ítems con una media de respuesta más baja son el $20(M=0,04)$, el $6(M=0,06)$ y el $19(M=0,08)$. La desviación típica de todos los ítems oscila entre el 0,207 del ítem 20 y el 0,502 de los ítems 3 y 11 .

\section{Evidencias de validez}

Estructura factorial. Inicialmente se realizó un Análisis Factorial Confirmatorio para verificar la estructura encontrada en la validación previa realizada por Martin et al. (2006). Los resultados indican que una estructura de tres factores no es psicométricamente apropiada.

Por ello, y con el fin de encontrar una solución factorial adecuada, se procede a realizar un Análisis Factorial Exploratorio, utilizando como matriz de entrada una matriz de correlaciones de Pearson. En dicho análisis se obtienen evidencias de una estructura esencialmente unidimensional como la más adecuada. Posteriormente, se lleva a cabo un Análisis Factorial Confirmatorio utilizando únicamente aquellos cuestionarios con una puntuación global superior a 0 . Diversos indices confirman que la estructura unidimensional resulta adecuada para el test (Hu y Bentler, 1999; Jackson, 2007; Kline, 2005), entre ellos la relación entre $\chi^{2}$ y los grados de libertad apuntan a un ajuste muy aceptable con una puntuación por debajo de 2 (CMIN/DF = 1,101), al igual que el Índice Comparativo de Ajuste, en torno a 0,95 (CFI =0,947), y la raíz cuadrada media del error de aproximación, que se mantiene por debajo de 0,6 (RMSEA =0,030). El Intervalo de Confianza del RMSEA es de 0,30 (IC entre 0,000 y 0,049), un valor menor al del modelo independiente. De la misma forma,
Tabla 2. Estadísticos descriptivos de los items del CPQ-A.

\begin{tabular}{|c|c|c|c|c|}
\hline İtem & Media & Desv. tip. & Asimetria & Curtosis \\
\hline 1 & 0,29 & 0,458 & 0,913 & $-1,188$ \\
\hline 2 & 0,16 & 0,369 & 1,873 & 1,534 \\
\hline 3 & 0,49 & 0,502 & 0,036 & $-2,035$ \\
\hline 4 & 0,14 & 0,351 & 2,069 & 2,322 \\
\hline 5 & 0,15 & 0,360 & 1,967 & 1,904 \\
\hline 6 & 0,06 & 0,243 & 3,664 & 11,633 \\
\hline 7 & 0,36 & 0,481 & 0,604 & $-1,665$ \\
\hline 8 & 0,28 & 0,449 & 1,011 &,- 995 \\
\hline 9 & 0,18 & 0,385 & 1,701 & 0,911 \\
\hline 10 & 0,13 & 0,332 & 2,299 & 3,343 \\
\hline 11 & 0,47 & 0,502 & 0,109 & $-2,025$ \\
\hline 12 & 0,10 & 0,299 & 2,737 & 5,590 \\
\hline 13 & 0,31 & 0,466 & 0,820 & $-1,352$ \\
\hline 14 & 0,31 & 0,466 & 0,820 & $-1,352$ \\
\hline 15 & 0,28 & 0,449 & 1,011 &,- 995 \\
\hline 16 & 0,20 & 0,399 & 1,549 & 0,406 \\
\hline 17 & 0,19 & 0,392 & 1,623 & 0,646 \\
\hline 18 & 0,22 & 0,418 & 1,348 &,- 188 \\
\hline 19 & 0,08 & 0,273 & 3,129 & 7,935 \\
\hline 20 & 0,04 & 0,207 & 4,470 & 18,307 \\
\hline 21 & 0,26 & 0,440 & 1,116 &,- 769 \\
\hline 22 & 0,07 & 0,259 & 3,374 & 9,551 \\
\hline 23 & 0,15 & 0,360 & 1,967 & 1,904 \\
\hline 24 & 0,23 & 0,424 & 1,286 &,- 352 \\
\hline 25 & 0,23 & 0,424 & 1,286 &,- 352 \\
\hline 26 & 0,38 & 0,489 & 0,484 & $-1,798$ \\
\hline 27 & 0,13 & 0,342 & 2,179 & 2,798 \\
\hline
\end{tabular}

Tabla 3. Pesos factoriales de los items del CPQ-A.

\begin{tabular}{cccccc}
\hline No item & Peso Factorial & № item & Peso Factorial & № item & Peso Factorial \\
\hline 27 &, 508 & 18 &, 409 & 9 &, 526 \\
26 &, 406 & 17 &, 208 & 8 &, 506 \\
25 &, 314 & 16 &, 306 & 7 &, 369 \\
24 &, 282 & 15 &, 495 & 6 &, 528 \\
23 &, 462 & 14 &, 244 & 5 &, 400 \\
22 &, 518 & 13 &, 401 & 4 &, 248 \\
21 &, 472 & 12 &, 081 & 3 &, 224 \\
20 &, 223 & 11 &, 028 & 2 &, 192 \\
19 &, 336 & 10 &, 247 & 1 &, 181 \\
\hline
\end{tabular}


el valor del AIC es igual a 554,737, también menor que el de los modelos saturado e independiente. El valor del parámetro de no centralidad (NCP $=28,737)$ también es lo suficientemente bajo como para aceptar el modelo unidimensional y el Índice Esperado de Valoración Cruzada (ECVI $=4,998$ ) apunta a un buen ajuste de este modelo, al ser menor que el obtenido para el modelo saturado $(E C V I=7,297)$ e independiente $(9,014)$. Los pesos factoriales obtenidos para cada uno de los items pueden observarse en la Tabla 3. Tan sólo los ítems 1, 2, 11 y 12 obtienen pesos factoriales por debajo de 0,20.

Validez predictiva. La correlación de Pearson entre la puntuación en el CPQ-A y la intensidad de consumo en el último mes es de 0,546 ( $p<0,001)$. Este resultado indica que a mayor puntuación en el cuestionario, mayor intensidad de consumo.

El resultado de la regresión logística de la puntuación del CPO-A sobre el consumo elevado y moderado de cannabis en el último mes arroja una odds ratio de 1,312 ( $p<0,001$; IC $95 \%=1,181-1,457)$.

La correlación de Pearson entre la puntuación directa total del CPO-A y la del CAST es de 0,582 ( $p<0,001)$. Ambas pruebas parecen estar evaluando un mismo constructo, aunque existen no obstante diferencias entre los dos instrumentos, dado que la varianza explicada común es del $36 \%$.

La correlación entre el grado de preocupación general ocasionado por el consumo del cannabis y la puntuación directa total del CPO-A es de 0,348 ( $p<0,001)$. Si se analiza la correlación entre el número de áreas que son objeto de preocupación y la puntuación del CPQ-A, el resultado que se obtiene es $0,371(p<0,001)$. Ambas correlaciones indican que hay relación entre las puntuaciones del CPO-A y la preocupación generada a los usuarios por el consumo de cannabis. Una mayor puntuación en el CPQ-A hace más probable que nos encontremos con una preocupación global superior y un mayor número de áreas de la vida afectadas por dicha preocupación.

En relación con los criterios diagnósticos del DSM IV $T R$, la regresión logística binaria muestra que la puntuación en el CPO-A explica el 50,8\% de la varianza en el caso de la dependencia y el $36,2 \%$ en el del abuso ( $R$ cuadrado de Nagelkerke), clasificando correctamente al $84,7 \%$ de los casos de dependencia, y al 77,8\% de los de abuso. En el caso de la dependencia, la odds ratio (ExpB) de la puntuación en el CPO-A es de 1,545, y en el del abuso, de 1,368.

El punto de corte óptimo según la curva ROC para la dependencia es de 5,5 con una sensibilidad del $80,4 \%$ y una especificidad del 86,7\%. En el caso del abuso, el punto de corte óptimo es de 4,5 con una sensibilidad de $76,5 \%$ y una especificidad de 76,3\%.

Las puntuaciones en el CPO-A permiten diferenciar a los jóvenes que muestran mayor malestar psicopatológico según el BSI. De acuerdo con los resultados de las pruebas t y con un punto de corte de 5,5 en las puntuaciones del CPQ, se encuentran diferencias estadísticamente significativas $(p<0,05)$ en todas las escalas del BSI, siendo siempre superior la puntuación obtenida por aquellos participantes cuya puntuación supera el punto de corte (ver Tabla 4). Según la d de Cohen, todas las diferencias presentan un tamaño del efecto medio 0 alto.

Tabla 4.- Diferencias en las puntuaciones de las subescalas del BSI para aquellos participantes con puntuaciones superiores e inferiores al punto de corte del CPQ-A de 5,5 puntos.

\begin{tabular}{lcccc}
\hline Subescalas del BSI & t de Student & gl & $\begin{array}{c}\text { Sig. } \\
(2 \text { colas })\end{array}$ & d de Cohen \\
\hline Sintomas de somatización & $-3,989$ & 142 & $<0,001$ & 0,70 \\
$\begin{array}{l}\text { Sintomas obsesivo - compulsivos } \\
\text { Sintomas de sensibilidad }\end{array}$ & $-3,173$ & 75,978 & 0,002 & 0,61 \\
interpersonal & $-3,625$ & 72,858 & 0,001 & 0,71 \\
Sintomas depresivos & $-5,059$ & 72,036 & $<0,001$ & 1,00 \\
Sintomas de ansiedad & $-4,109$ & 74,905 & $<0,001$ & 0,79 \\
Sintomas de hostilidad & $-4,315$ & 61,643 & $<0,001$ & 0,91 \\
Sintomas fóbicos & $-3,183$ & 79,349 & 0,002 & 0,60 \\
Sintomas paranoides & $-3,090$ & 79,350 & 0,003 & 0,58 \\
Sintomas de psicoticismo & $-3,727$ & 79,966 & $<0,001$ & 0,70 \\
Sintomas adicionales & $-2,469$ & 67,719 & 0,016 & 0,50 \\
Indice de severidad global & $-4,299$ & 72,013 & $<0,001$ & 0,85 \\
Total de sintomas positivos & $-5,486$ & 142 & $<0,001$ & 0,96 \\
Indice de malestar por sintomas & $-3,516$ & 142 & $<0,001$ & 0,61 \\
\hline positivos & & & & \\
\hline
\end{tabular}

\section{Fiabilidad}

La fiabilidad del CPO-A se estimó mediante la fórmula del Alfa de Cronbach, obteniéndose un 0,86 para el total del cuestionario, y el coeficiente de fiabilidad estimado por el método de las dos mitades aleatorias, que resultó igual a 0,87 . Los Índices de Discriminación se mantienen por encima de 0,20, a excepción de los ítems $1,2,11$ y 12 . La mayor correlación ítem-total corresponde al ítem 22 (i.d. = 0,46).

\section{Discusión}

Los datos comparativos entre la muestra de este estudio y los obtenidos de la última encuesta nacional ESTUDES del Plan Nacional sobre Drogas (Observatorio Español sobre Drogas, 2009), indican que los jóvenes de la muestra actual tienen prevalencias de consumo de cannabis ligeramente más altas. Las mayores tasas de consumo de cannabis pueden deberse a las diferencias en el rango de edad de ambos estudios, con unos límites superiores en la presente investigación. De la misma forma, el procedimiento de obtención de la muestra también es diferente, ya que en este caso se buscó maximizar el número de consumidores de cannabis. Únicamente las prevalencias de consumo de cannabis en el último mes entre las mujeres son ligeramente inferiores en nuestros resultados en comparación con la encuesta nacional ESTUDES. En todo caso, en ambas muestras es más probable que los consumidores de cannabis sean varones. Las diferencias entre las cifras de prevalencia vida, año y mes de ambos estudios no son relevantes y no parece encontrarse por tanto ningún dato que impida la generalización a la población española de los resultados obtenidos. 
Los análisis factoriales exploratorios y confirmatorios apuntan a una estructura esencialmente unidimensional, que no coincide con la estructura trifactorial apuntada por el estudio australiano (Martin et al., 2006). Esta divergencia puede ser debida a las diferencias en la población evaluada. En nuestro estudio se recurrió a una muestra de jóvenes escolarizados consumidores de cannabis con el propósito de validar la capacidad de screening del instrumento. Por ello el único criterio de inclusión era el consumo reciente de cannabis (cualquier uso durante el último mes). Sin embargo, en la validación australiana la muestra es de conveniencia, evaluando únicamente a jóvenes que acuden voluntariamente al estudio tras experimentar problemas con el cannabis. Debido a la diferente procedencia, nuestra muestra de consumidores de cannabis que ha cumplimentado el CPO-A no está balanceada por sexos y está compuesta de un 71,5\% de chicos y un $28,5 \%$ de chicas, lo que refleja la mayor prevalencia masculina que se encuentra en las encuestas de consumo. Por otra parte, la edad media en la muestra española es de 17,1 años (DT = 1,17), algo superior a los $16,1(\mathrm{DT}=1,1)$ de la muestra utilizada para la validación australiana. En cuanto al uso de cannabis, nuestra muestra parece tener un consumo menos severo, ya que se inició a los 15,2 años, frente a los 13,7 del otro estudio, y tan sólo un 22,9\% informa tener un consumo diario, frente al $31 \%$ que se obtiene en el trabajo anterior. Asimismo, el porcentaje de jóvenes con diagnóstico de dependencia es inferior en nuestro estudio $(31,9 \%$ de la muestra española frente al $46 \%$ de la australiana), y tan sólo un $6,9 \%$ de nuestros participantes refería haber considerado ir a tratamiento (resultados no publicados), frente al 23\% de la validación previa.

Es posible que una menor severidad del consumo de cannabis (menor frecuencia e historia de uso) conlleve la presencia de perfiles menos graves en cada uno de los factores del cuestionario (consecuencias psicológicas, físicas y negativas agudas). Esto derivaría en un cuadro general en el que todas las áreas se encuentran afectadas, sin que ninguna de ellas destaque de forma significativa sobre las demás. La consecuencia es que el cuestionario presenta, en la población general española de jóvenes consumidores, una mayor adecuación a una estructura unidimensional.

Las evidencias de validez predictiva concurrente del CPO-A son notables. Existe una fuerte correlación entre el CPQ-A y el consumo de cannabis durante el último mes. El CPO-A presenta un buen valor predictivo a la hora de discriminar entre consumidores con moderada y elevada frecuencia de consumo en el último mes, con un ratio de probabilidad igual a 1,312.

También se encuentra una fuerte correlación entre el CPQ-A y el CAST, con un 36\% de varianza común. El margen de varianza no compartida puede deberse a: 1) que el CAST evalúa los problemas experimentados en el último año, mientras el CPQ-A se limita a los últimos 3 meses; y 2) el CAST se orienta a la detección diagnóstica de abuso mientras que el CPQ-A explora más ampliamente diversos problemas psicosociales relacionados con el uso habitual del cannabis.

Se ha encontrado también relación entre la puntuación obtenida en el CPQ-A y la preocupación del consumidor por las consecuencias del consumo de cannabis. Esto supone una validación de la selección de ítems del CPQ-A como un conjunto de problemas clínicamente significativos según los propios consumidores. Si el cuestionario indica un consumo más problemático, existe una mayor probabilidad de que el participante exprese mayor preocupación por el consumo y que señale más ámbitos de su vida como objeto de preocupación.

EI CPQ-A ofrece una sensibilidad y especificidad adecuadas en la detección del abuso y la dependencia según los criterios del DSM-IV-TR. Aunque se discute la idoneidad de utilizar criterios diagnósticos de abuso y dependencia con adolescentes (Hartman et al., 2008), la presencia de estos trastornos es indicativo de un consumo problemático detectado por el cuestionario. El CPO-A ofrece una herramienta fiable y válida para ayudar en la detección de estos trastornos en población joven.

Por último, y en consonancia con los hallazgos científicos que relacionan el consumo de cannabis con una mayor probabilidad de presentar alteraciones psicopatológicas (Hides et al., 2009; Kaminer, Connor, y Curry, 2008; Rubino et al., 2011; Stefanis et al., 2004), una puntuación superior a 5,5 en el CPQ-A parece estar relacionada con una mayor presencia de síntomas psicopatológicos, entre los que predominan los síntomas depresivos, de hostilidad y de ansiedad, de acuerdo con los resultados obtenidos en el BSI.

En el contraste entre los dos instrumentos utilizados en el presente estudio se encuentra que el CPQ-A ofrece una fiabilidad superior al CAST, con un Alfa de Cronbach de 0,86 en el primero frente al 0,72 del CAST (Klempova et al., 2009). Cuatro ítems del CPQ-A tienen no obstante un índice de discriminación inferior a 0,20 y presentan un bajo peso factorial, lo que unido al considerable número de items que componen la escala sugiere una reducción del instrumento con el fin de facilitar su utilidad de screening. En efecto, una limitación del CPO-A es su mayor extensión, que requiere un mayor tiempo de cumplimentación. En este sentido, Proudfoot et al. (2010) han desarrollado recientemente una versión reducida de 12 ítems, el CPQ-A-S, adaptado a población australiana, con una Alfa de Cronbach de 0,73. Las propiedades psicométricas y utilidad clínica de dicha prueba no han sido aún analizadas en población española.

\section{Limitaciones}

El presente estudio tiene no obstante algunas limitaciones susceptibles de ser subsanadas en estudios posteriores. La muestra obtenida está circunscrita a una sola región de España y no se ha seguido un procedimiento de captación aleatorizado. La representatividad de la muestra podría por tanto mejorar recurriendo a una muestra territorial más amplia y a un método de extracción de participantes aleatorio. Sin embargo, tal y como se ha explicado más arriba, el contraste de los principales parámetros sobre consumo de la muestra de este estudio con otros datos nacionales no detecta sesgos que invaliden los resultados obtenidos.

Algunos aspectos como la estabilidad temporal de las puntuaciones o la capacidad predictiva de la prueba no pueden responderse con un diseño transversal. Tampoco es 
posible extraer conclusiones firmes sobre las razones que subyacen a la discrepancia en la solución factorial encontrada en contraste con el estudio de Martin y cols. (2006). Estas cuestiones sólo pueden resolverse con nuevas aplicaciones en muestras más amplias y con diseños de carácter longitudinal.

Aunque el tiempo de aplicación de la prueba no supera los 5 minutos, los resultados del presente estudio confirman investigaciones recientes que sugieren que el CPQ-A puede beneficiarse de una reducción del número de ítems que lo conforman, al no aportar información adicional ni contribuir de forma significativa a la mejora de las propiedades psicométricas. El trabajo de Proudfoot et al. (2010) acorta la prueba hasta los 12 items, con una leve reducción de las buenas propiedades psicométricas del test.

En conclusión, el CPQ-A resulta ser un instrumento fiable y válido, con un gran potencial como herramienta de screening así como de apoyo a la evaluación clínica, con un acercamiento a diversas áreas afectadas por el consumo habitual de cannabis, y consideradas significativas por los propios consumidores. Los resultados de esta investigación sugieren que se ha de continuar analizando las propiedades psicométricas del CPQ-A con otros diseños de investigación, las diferencias entre el CPQ-A y el CAST en cuanto a su utilidad de screening y ayuda en el diagnóstico clínico, asi como las características de la versión reducida, el CPO-A-S.

\section{Reconocimientos}

Este estudio forma parte de una investigación financiada por el Ministerio de Innovación y Ciencia de España (Ref: MICINN-08-PSI2008.00309). Los autores agradecen la colaboración de la Consejería de Educación del Principado de Asturias así como de las escuelas participantes, su personal y sus alumnos.

\section{Conflicto de intereses}

Los autores del artículo manifiestan no tener ningún conflicto de intereses.

\section{Referencias}

Adamson, S. J., Kay-Lambkin, F. J., Baker, A. L., Lewin, T. J., Thornton, L., Kelly, B. J. y Sellman, J. D. (2010). An improved brief measure of cannabis misuse: the Cannabis Use Disorders Identification TestRevised (CUDIT-R). Drug and A/cohol Dependence, 110, 137-143.

American Psychiatric Association. (2002). DSM-IV TR - Manual Estadístico y Diagnóstico de los Trastornos Mentales. Texto Revisado. Barcelona: Masson.

Arias Horcajadas, F. (2007). Revisión sobre el consumo de cannabis como factor de riesgo de la esquizofrenia. Adicciones, 19, 191-204.

Baño Rodrigo, M. D., Ramos Atance, J. A., López García, M. L., de Antonio Pérez, V. y Guillén Llera, J. L. (2004). Sindrome de abstinencia y otros efectos en jóvenes fumadores de hachís. Adicciones, 16, 19-30.

Caldeira, K. M., Kasperski, S. J., Sharma, E., Vincent, K. B., O'Grady, K. E., Wish, E. D. y Arria, A. M. (2009). College students rarely seek help despite serious substance use problems. Journal of Substance Abuse Treatment, 37, 368-378.

Copeland, J., Gilmour, S., Gates, P. y Swift, W. (2005). The Cannabis Problems Questionnaire: factor structure, reliability, and validity. Drug and Alcohol Dependence, 80, 313-319.

Crean, R. D., Crane, N. A. y Mason, B. J. (2011). An evidence based review of acute and long-term effects of cannabis use on executive cognitive functions. Journal of Addiction Medicine, 5, 1-8.

Derogatis, L. R. y Melisaratos, N. (1983). The Brief Symptom Inventory: An introductory report. Psychological Medicine, 13, 595-605.

ESPAD Project. (2009). ESPAD - The European School Survey Project on Alcohol and Other Drugs. 2009. Recuperado de http://www. espad.org

European Monitoring Centre for Drugs and Drug Addiction. (2011). Table GPS-10. Frequency of use of cannabis amongst users in the last 30 days in national general population surveys. Recuperado de http://www.emcdda.europa.eu/stats11/gpstab10a

Fernández-Artamendi, S., Fernández-Hermida, J. R., Secades-Villa, R. y Garcia-Portilla, P. (2011). Cannabis y Salud Mental. Actas Españolas de Psiquiatría, 39, 180-190.

Fonseca-Pedrero, E., Paino-Piñeiro, M., Lemos-Giráldez, S., VillazónGarcía, U. y Muñiz, J. (2009). Validation of the Schizotypal Personality Questionnaire-Brief Form in adolescents. Schizophrenia Research, 111, 53-60.

Gossop, M., Darke, S., Griffiths, P., Hando, J., Powis, B., Hall, W. y Strang, J. (1995). The Severity of Dependence Scale (SDS): psychometric properties of the SDS in English and Australian samples of heroin, cocaine and amphetamine users. Addiction, 90,607-614.

Hall, W. y Degenhardt, L. (2009). Adverse health effects of nonmedical cannabis use. Lancet, 374, 1383-1391.

Hartman, C. A., Gelhorn, H., Crowley, T. J., Sakai, J. T., Stallings, M. y Young, S. E. (2008). Item Response Theory Analysis of DSM-IV Cannabis Abuse and Dependence Criteria in Adolescents. Journal of the American Academy of Child \&t Adolescent Psychiatry, 47, 165-173. doi: 10.1097/chi.0b013e31815cd9f2

Hides, L., Lubman, D. I., Buckby, J., Yuen, H. P., Cosgrave, E., Baker, K. y Yung, A.R. (2009). The association between early cannabis use and psychotic-like experiences in a community adolescent sample. Schizophrenia Research, 112, 130-135.

Hu, L. T. y Bentler, P. M. (1999). Cutoff criteria for fit indices in covariance structure analysis: Conventional criteria versus new alternatives. Structural Equation Modeling: A Multidisciplinary Journal, 6, 1-55.

Jackson, D. L. (2007). The effect of the number of observations per parameter in misspecified confirmatory factor analytic models. Structural Equation Modeling: A Multidisciplinary Journal, 14, 48-76.

Kaminer, Y., Connor, D. F. y Curry, J. F. (2008). Treatment of comorbid adolescent cannabis use and major depressive disorder. Psychiatry (Edgmont), 5, 34-39.

Kessler, R. C., Aguilar-Gaxiola, S., Berglund, P. A., Caraveo-Anduaga, J. J., DeWit, D. J., Greenfield, S. F., ... y Vega, W.A. (2001). Patterns and predictors of treatment seeking after onset of a substance use disorder. Archives of General Psychiatry, 58, 1065-1071.

Klempova, D., Sánchez, A., Vicente, J., Barrio, G., Domingo, A., Suelves, J. M., ... y Ramírez, V. (2009). Consumo problemático de cannabis en estudiantes españoles de 14 a 18 años: Validación de escalas. Madrid: Delegación del Gobierno para el Plan Nacional sobre Drogas. Ministerio de Sanidad y Politica Social. Centro de Publicaciones. 
Kline, R. B. (2005). Principles and practice of structural equation modeling ( $2^{\text {nd }}$ ed.). New York: The Guilford Press.

Legleye, S., Karila, L., Beck, F. y Reynaud, M. (2007). Validation of the CAST, a general population Cannabis Abuse Screening Test. Journal of Substance Use, 12, 233-242.

Martin, G., Copeland, J., Gilmour, S., Gates, P. y Swift, W. (2006). The Adolescent Cannabis Problems Questionnaire (CPO-A): psychometric properties. Addictive Behaviors, 31, 2238-2248.

Muñiz-Fernández, J. y Hambleton, R. K. (1996). Directrices para la traducción y adaptación de los tests. Papeles del Psicólogo, 66, 63-70.

Observatorio Español sobre Drogas. (2009). Informe 2009 del Observatorio Español sobre Drogas. Situación y tendencias de los problemas de drogas en España. Madrid: Ministerio de Sanidad y Política Social. Secretaria General de Política Social y Consumo. Delegación del Gobierno para el Plan Nacional sobre Drogas.

Parolaro, D. (2010). Adolescent cannabis consumption and schizophrenia: evidencias empíricas y experimentales. Adicciones, 22 (3), 185-190.
Pereda, N., Forns, M. y Pero, M. (2007). Dimensional structure of the brief symptom inventory with Spanish college students. Psicothema, 19 (4), 634-639.

Piontek, D., Kraus, L. y Klempova, D. (2008). Short scales to assess cannabis-related problems: a review of psychometric properties. Substance Abuse Treatment, Prevention and Policy, 3: 25.

Proudfoot, H., Vogl, L., Swift, W., Martin, G. y Copeland, J. (2010). Development of a short cannabis problems questionnaire for adolescents in the community. Addictive Behaviors 35, 734-737.

Rubino, T., Zamberletti, E. y Parolaro, D. (2011). Adolescent exposure to cannabis as a risk factor for psychiatric disorders. Journal of Psychopharmacology. Avance de publicación online. doi: 10.1177/0269881111405362

Stefanis, N. C., Delespaul, P., Henquet, C., Bakoula, C., Stefanis, C. N. y Van Os, J. (2004). Early adolescent cannabis exposure and positive and negative dimensions of psychosis. Addiction, 99, 1333-1341.

Substance Abuse and Mental Health Services Administration. (2010). Results from the 2009 National Survey on Drug Use and Health: Volume I. Summary of National Findings. Rockville, MD.

\section{ANEXO 1}

\section{(CPO-A) CUESTIONARIO DE PROBLEMAS ASOCIADOS AL CANNABIS PARA ADOLESCENTES}

1. A continuación figuran una serie de problemas que a menudo experimentan las personas que consumen cannabis.

2. Por favor, lee atentamente cada pregunta e indícanos si en los últimos 3 meses has experimentado alguna de estas

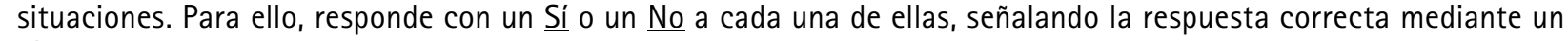
círculo o una $\mathrm{X}$ en la columna correspondiente.

\section{En los últimos 3 meses...}

\begin{tabular}{|c|c|c|}
\hline & No & Si \\
\hline \multicolumn{3}{|l|}{ 1. ¿Has fumado cannabis con más frecuencia que antes estando solo? } \\
\hline \multicolumn{3}{|l|}{ 2. ¿Te ha preocupado estar con gente que no conoces cuando estabas "colocado"? } \\
\hline \multicolumn{3}{|l|}{ 3. ¿Has pasado más tiempo con amigos que fuman cannabis que con otros amigos? } \\
\hline \multicolumn{3}{|l|}{ 4. ¿Te han criticado tus amigos por fumar cannabis en exceso? } \\
\hline \multicolumn{3}{|l|}{ 5. ¿Has contraido alguna deuda por consumir cannabis? } \\
\hline \multicolumn{3}{|l|}{ 6. ¿Has empeñado alguna de tus pertenencias para comprar cannabis? } \\
\hline \multicolumn{3}{|l|}{ 7. ¿Has tenido que poner excusas sobre tu gasto de dinero? } \\
\hline \multicolumn{3}{|l|}{ 8. ¿Te ha preocupado la cantidad de dinero que has estado gastando en cannabis? } \\
\hline \multicolumn{3}{|l|}{ 9. ¿Te han pillado mintiendo sobre el dinero que gastas? } \\
\hline \multicolumn{3}{|l|}{ 10. ¿Has tenido problemas con la policia debido a tu consumo de cannabis? } \\
\hline \multicolumn{3}{|l|}{ 11. ¿Te has encontrado físicamente mal tras haber fumado cannabis? } \\
\hline \multicolumn{3}{|l|}{ 12. ¿Te has desmayado alguna vez después de haber fumado cannabis? } \\
\hline \multicolumn{3}{|c|}{ 13. ¿Has sentido dolor en el pecho o en los pulmones tras haber estado fumando cannabis? } \\
\hline \multicolumn{3}{|l|}{ 14. ¿Has tenido bronquitis o tos persistente? } \\
\hline \multicolumn{3}{|l|}{ 15. ¿Te has sentido paranoico o antisocial tras haber estado fumando cannabis? } \\
\hline \multicolumn{3}{|l|}{ 16. ¿Has perdido peso sin habértelo propuesto? } \\
\hline \multicolumn{3}{|l|}{ 17. ¿Te has descuidado fisicamente? } \\
\hline \multicolumn{3}{|l|}{ 18. ¿Te has sentido deprimido durante más de una semana? } \\
\hline \multicolumn{3}{|l|}{ 19. ¿Te has sentido tan deprimido como para pensar en suicidarte? } \\
\hline \multicolumn{3}{|c|}{ 20. ¿Has dejado de hacer alguna actividad de la que antes disfrutabas por tu consumo de cannabis? } \\
\hline \multicolumn{3}{|l|}{ 21. ¿Te has sentido con menos energia de lo habitual? } \\
\hline \multicolumn{3}{|l|}{ 22. ¿Te ha resultado dificil disfrutar como siempre de tus aficiones habituales? } \\
\hline \multicolumn{3}{|l|}{ 23. ¿Tu salud general ha estado peor de lo habitual? } \\
\hline \multicolumn{3}{|l|}{ 24. ¿Te ha preocupado perder el contacto con amigos o familiares? } \\
\hline \multicolumn{3}{|l|}{ 25. ¿Te ha preocupado la falta de motivación para hacer cosas? } \\
\hline \multicolumn{3}{|l|}{ 26. ¿Te ha resultado más dificil de lo habitual concentrarte? } \\
\hline 27. ¿Has estado preocupado por sentimientos de aislamiento o de desapego? & & \\
\hline
\end{tabular}


